\title{
Editorial
}

\section{DOES THERE EXIST A SUBGROUP OF MB PATIENTS AT GREATER RISK OF RELAPSE AFTER MDT?}

It has been reported that more than 10 million leprosy patients have been cured by multidrug therapy (MDT), ${ }^{1}$ but the definition of cure is vague. Because the clinical, bacteriological and immunological manifestations of leprosy show such wide variation, the requirements of chemotherapy are different for patients at various positions on the spectrum, and completion of MDT may or may not be equivalent to cure of the disease. Apparently, the long-term efficacy of MDT should be assessed by more solid data. For patients with multibacillary (MB) leprosy, routine clinical examination and skin smears are not sensitive enough to monitor the long-term efficacy of chemotherapy; while mouse footpad inoculation can quantify the initial 99.999\% killing of Mycobacterium leprae ${ }^{2-4}$ an advanced lepromatous patient may begin treatment with a total of $10^{10}$ to $10^{11}$ viable organisms, and the failure to demonstrate multiplication of $M$. leprae at the end of treatment even in nude mice may not be taken as evidence that all viable $M$. leprae within the host have been killed. Therefore, the only way to assess the long-term efficacy of chemotherapy among MB patients is to measure the relapse rate after cessation of treatment, as is the case for other infectious diseases.

We reported in 1992 that only a single relapse was detected among $35 \mathrm{MB}$ patients who had been treated with 24 months of MDT, with an overall relapse rate of $2.9 \%$, or 0.8 per 100 patient-years, being the lowest relapse rate among the various rifampicin (RMP)-containing combined regimens that had been tested at Institut Marchoux, Bamako, Mali. ${ }^{5}$ Because the mean duration of follow-up for this particular group of patients was only $41.9 \pm 12.1$ months after stopping MDT, we emphasized that it was only a provisional observation, and the patients should continue to be followed up. ${ }^{5}$ During an additional 2.5 years of follow up, six more relapses were detected among this cohort, and the overall relapse rate had increased to $20 \%$, or 3.3 per 100 patient-years. $^{6}$ The mean incubation period, i.e. the mean interval between stopping treatment and the appearance of relapse, of the seven relapses was $62.7 \pm 18.7$ months, which did not differ significantly from the mean incubation period of $58.4 \pm 25.1$ months among 68 relapses after stopping treatment with other RMPcontaining combined regimens, ${ }^{5}$ indicating that relapses after MDT also occurred late. All seven relapses were observed among the 18 patients who had an initial bacterial index (BI), i.e. average $\mathrm{BI}$ before MDT, of $\geq 4.0$, whereas no relapse was detected among the 17 patients whose initial BI was $<4.0{ }^{6}$ This indicates a close correlation between relapse and the size of the bacterial population in patients before MDT, and a high initial BI of $\geq 4.0$ appeared to be the most important risk factor for relapse among patients with MB leprosy. However, because the sample size was small, the unacceptably high relapse rate among this cohort in general, 
and among those patients with an initial $\mathrm{BI}$ of $\geq 4.0$ in particular, must be interpreted with caution.

Recently, our observations of a high relapse rate among MB patients after MDT and its close correlation with high initial BI were confirmed by Girdhar et al. of the Central JALMA Institute, India, who studied a larger number of patients. ${ }^{7}$ After a mean duration of follow-up of 3.7 years among $260 \mathrm{MB}$ patients who had received treatment with 24-month MDT, 20 patients relapsed, with an overall relapse rate of $7.7 \%$, or 2.04 per 100 patient-years. The relapse rate was $16.8 \%(18 / 107)$ or 4.29 per 100 patient-years among patients with an initial $\mathrm{BI}$ of $\geq 4.0$, many times greater than the relapse rate of $1.3 \%(2 / 153)$ or 0.36 per 100 patientyears among those with an initial BI of $<4.0,{ }^{7}$ but smaller than the corresponding figures of $38.9 \%$ or 7.0 per 100 patient-years among patients with initial BI of $\geq 4.0$ at Institut Marchoux. ${ }^{6}$ Most likely, the latter difference resulted from the relatively short duration of follow-up at JALMA, as pointed out by the authors. ${ }^{7}$ Among another cohort of 301 patients at JALMA, despite treatment with MDT until skin smear negativity (mean duration $4.9 \pm 2.3$ years), 12 patients relapsed after a mean duration of 3.6 years of follow-up, with an overall relapse rate of $3.99 \%$, or 1.11 per 100 patient-years; 11 of the 12 relapses occurred among patients who had an initial $\mathrm{BI}$ of $\geq 4.0$. The relapse rate of 1.27 per 100 patient-years among this subgroup was significantly smaller than the relapse rate of 4.29 per 100 patient-years among the corresponding subgroup of patients treated with 24 -month MDT. ${ }^{7}$ The important difference between the results from JALMA and ours is that the relapses at JALMA occurred earlier, most of them were recorded during the first 3 years after stopping treatment (the mean durations of follow-up of the two cohorts at JALMA were only slightly longer than 3 years). A possible explanation of this difference is that relapse was diagnosed at JALMA when there was an increase in $\mathrm{BI}$ of $\geq 2+$ over the previous value from any single site, with or without the appearance of new lesions, ${ }^{7}$ whereas we diagnosed relapse only when the increase of BI was accompanied by reactivation of pre-existing lesions or the occurrence of definite new lesions, which often appeared after the increase in BI. ${ }^{5,6}$

The results from Institut Marchoux and from JALMA clearly suggest the existence of a high risk subgroup of MB patients who show a strong tendency of relapse after MDT. Should this subgroup be large, the strategy for leprosy elimination and the policy of chemotherapy for MB leprosy should be reviewed and, perhaps, revised. That the relapse rate among $\mathrm{MB}$ patients with initial $\mathrm{BI}$ of $<4.0$, who represent the great majority of $\mathrm{MB}$ patients in the field, was low could explain the low relapse rate in the field, even after a longer duration of follow up. ${ }^{8-10}$

However, the conclusions of others have been very different from that based on the data from Institut Marchoux and of JALMA. No relapse was detected among 34 previously untreated MB patients with initial BI of $\geq 3.0$ who had been followed up at the Schieffelin Leprosy Research and Training Centre, Karigiri, India, for more than 4 years after completion of 24-month MDT. ${ }^{11}$ Recently, the same institute reported only a single relapse among 46 patients, including the previous 34 cases, after $9.26 \pm 2.98$ years of follow-up. ${ }^{12}$ Although the relapse rate was very low, it should be emphasized that the definition of high initial BI at Karigiri was $\geq 3$.0, and that the mean value of the average BI of the patients in this group was only $3.4,{ }^{11}$ indicating that the bacterial load of these patients as a group was significantly lower than those designated as patients with high initial BI at Institut Marchoux ${ }^{5,6}$ or at JALMA. ${ }^{7}$ Gebre et al. from ALERT, Addis Ababa, Ethiopia, reported recently that in the AMFES cohort not a single confirmed relapse was detected among $256 \mathrm{MB}$ patients who had been followed up for a mean duration of 4.3 years after completion of MDT, although 57 
patients of the cohort had an initial $\mathrm{BI}$ of $\geq 4.0$, and 20 of them had been followed-up for $\geq 5$ years. $^{13}$

Because there is no easy explanation for the deep disagreements regarding the risk of $\mathrm{MB}$ relapse after 24-month MDT and the existence of a high risk subgroup of MB patients who are prone to relapse, the only possible approach which may lead to reach a consensus is to collect more information from the long-term follow up of $\mathrm{MB}$ patients after completion of MDT. Although the 24-month MDT began to be tested in the late 1980s, it was officially recommended only in $1994^{14}$ and widely implemented thereafter; consequently, only a few reports of the results of follow-up after 24-month MDT have been published, and the durations of follow-up were, in general, relatively short. Nevertheless, the fact that millions of MB patients have completed their treatment with 24-month MDT suggests that unpublished results of long-term follow-up, carried out either by research institutes or by routine programmes, may be available; publication of these results may help to clarify the confusion and should therefore be encouraged. To facilitate a meaningful and valid comparison of results from different sources, the following principles are proposed:

1. The follow-up should involve a cohort of MB patients who had completed 24-month MDT within the stipulated 36 months. ${ }^{15}$ Here, MB leprosy refers to those who were clinically or histopathologically classified as lepromatous (LL), borderline lepromatous (BL), or mid-borderline (BB) by the Ridley-Jopling classification, or anyone who was BI positive at any site in the initial skin smears. For various reasons, the information published to date regarding the proportion of patients with an initial BI of $\geq 4.0$ among total caseload or total MB patients is very limited. Data from the AMFES cohort indicate that $9 \%$ of the total previously untreated patients, or $22 \%$ of their total MB case-load, had an initial $\mathrm{BI}$ of $\geq 4.0{ }^{13}$ these proportions seem unexpectedly high, and should be compared with data from elsewhere.

2. During follow-up, the patients should be examined both clinically and bacteriologically at regular intervals, preferably no less than once annually. The skin smears should be taken from the same four to six sites originally examined, and from any suspected new lesions. For those institutes or routine programs in which the patients were not followed up regularly, it may be useful to retrieve and examine a proportion of the MB patients 5 years or more after completion of 24-month MDT. The results may provide useful information regarding the risk of relapse among patients with MB leprosy.

3. Relapse was suspected if the BI at any site was found to have increased by at least $2+$ over the previous value, or if any new skin lesion was detected with a BI greater than that in any pre-existing lesion. Further examinations include repeating the clinical examination and skin smears before relapse is diagnosed. Although, in its early stage, relapse may occur in the absence of obvious new lesions, and for some investigators, the occurrence of new skin lesion has not been required for the diagnosis of relapse, ${ }^{7}$ skin lesions must occur sooner or later in real relapse, as either reactivated pre-existing lesions or entirely new lesions; also because the quality of skin smears is far below the desirable level in many control programs, we strongly recommend that relapse should be diagnosed on both clinical and bacteriological criteria: either a confirmed increase of the BI at any single site accompanied by reactivation of the pre-existing lesions, or the occurrence of definite new skin lesions with a BI higher than that in any pre-existing but non-reactivated lesions, or both. The demonstration of viable $M$. leprae by mouse footpad inoculation is helpful for confirmation of relapse, but the technique is expensive, time-consuming, and inaccessible 
to the great majority of routine programs. In addition, because we had demonstrated that viable $M$. leprae were presented in nearly $90 \%$ of relapses diagnosed on the basis of clinical examination and skin smears, ${ }^{5,6}$ mouse inoculation is not obligatory for the diagnosis of relapse.

Employing these procedures and criteria, the diagnosis of $\mathrm{MB}$ relapse is rather straightforward, unlike the diagnosis of PB relapse, which almost always requires differentiation from late reversal reaction. The risk of overdiagnosis of MB relapse does exist, but it is probably not very high if one follows strictly these procedures and criteria; on the other hand, the risk of underdiagnosis of $\mathrm{MB}$ relapse might be much greater, because of ignorance, poor quality of skin smears, insufficient duration of follow up, or a combination of all of these factors.

4. Because there was a clear tendency that more relapses were occurred with the extension of follow-up, also because the mean incubation period of relapse was at least $5 \pm 2$ years after treatment with 24-month MDT, ${ }^{5,6}$ each patient should be followed for at least 5 years, and preferably for 7 years.

A number of difficulties or constraints may be encountered in attempting to follow up MB patients after completion of MDT. First, in more and more routine programs, the patients were removed from the registration as soon as they have completed MDT, and, very often, their essential records, e.g. identity, address, previous examinations and history of treatment, are missing, resulting in difficulty to retrieve the right patients for follow-up. Second, because of integration of the vertical leprosy program into general health services, the responsibility for detection of the suspected relapse cases rests upon general health workers, but a significant proportion of whom do not possess the necessary skills; in addition, the general health services often lack the manpower and resources required to follow patients who have already completed their treatment with MDT, as they are no longer considered as 'cases'. 15 Finally, because of the poor quality of skin smears in the past, and because the skin smear service very often is no longer available in the field, it is difficult to identify the MB patients who comprise the high risk subgroup and, more important, to detect the suspects of relapse. If the actions needed to overcome these difficulties are not taken as soon as possible, confusion about the magnitude of the risk of $\mathrm{MB}$ relapse and the possible existence of high risk subgroup of MB patients who are prone to relapse after MDT is unlikely to be resolved.

MB relapse after 12-month MDT is another important topic, because, since 1998, the great majority of MB patients have been treated with MDT for only 12 months, and there is virtually no information about the relapse rate after the shorter duration of MDT treatment. Because both the definition of MB leprosy and duration of MDT have been changed, ${ }^{15}$ information about relapse after 12-month MDT must be collected separately from that with respect to 24-month MDT. If there exists a high-risk subgroup of MB patients who are prone to relapse after 24-month MDT, the relapse rate among patients in this subgroup may be even higher after 12-month MDT. On the other hand, relapse may nevertheless appear late, as had been demonstrated after treatment with various short course RMP-containing combined regimens. ${ }^{5}$

Finally, corticosteroids are being widely used for prevention and treatment of neuropathy, ${ }^{16,17}$ and the duration of corticosteroid treatment for new neuropathy in MB patients has been recommended to continue for at least 24 weeks. ${ }^{17}$ It remains unclear whether corticosteroid treatment may predispose to relapse of MB leprosy, or patients should be covered by chemotherapy during their treatment with corticosteroids. The experience from 
the AMFES cohort suggested that corticosteroid treatment does not predispose to MB relapse, and coverage by chemotherapy is not required; ${ }^{13}$ however, some investigators have thought otherwise. ${ }^{18}$ Apparently, there is no consensus, and more information should be collected to clarify the possible linkage between corticosteroid treatment and relapse among MB patients, particularly among those with a high initial BI.

\section{References}

${ }^{1}$ World Health Organization. Report on first meeting of the WHO Technical Advisory Group on elimination of leprosy. WHO/CDS/CPE/CEE/2000.4.

2 Grosset JH, Ji B, Guelpa-Lauras CC, Perani EG, N'Deli LN. Clinical trial of pefloxacin and ofloxacin in the treatment of lepromatous leprosy. Int J Lepr, 1990; 58: 281-295.

3 Ji B, Jamet P, Perani EC, Bobin P, Grosset JH. Powerful bactericidal activities of clarithromycin and minocycline against Mycobacterium leprae in lepromatous leprosy. J Infect Dis, 1993; 168: 188-190.

4 Ji B, Jamet P, Perani EG, Sow S et al. Bactericidal activity of single dose of clarithromycin plus minocycline, with or without ofloxacin, against Mycobacterium leprae in patients. Antimicrob Agents Chemother, 1996; 40: 2137-2141.

5 Marchoux Chemotherapy Study Group. Relapse in multibacillary leprosy patients after stopping treatment with rifampin-containing combined regimens. Int J Lepr, 1992; 60: 525-535.

6 Jamet P, Ji B, and the Marchoux Chemotherapy Study Group. Relapse after long-term follow up of multibacillary patients treated with WHO multidrug regimen. Int J Lepr, 1995; 63: 195-201.

7 Girdhar BK, Girdhar A, Kumar A. Relapses in multibacillary leprosy patients: effect of length of therapy. Lepr Rev, 2000; 71: 144-153.

${ }^{8}$ WHO Leprosy Unit. Risk of relapse in leprosy. WHO Document. WHO/CTD/LEP/94.1.

9 Li H-Y, Hu L-F, Huang W-B et al. Risk of relapse in leprosy after fixed-duration multidrug therapy. Int J Lepr, 1997; 65: 238-245.

10 Chen X-S, Li W-Z, Jiang C, Ye G-Y. Studies on risk of leprosy relapses in China: relapse after treatment with multidrug therapy. Int J Lepr, 1999; 67: 379-387.

11 Jesudasan K, Vijayakumaran P, Manimozhi N, Jayarajan T. Absence of relapse within 4 years among 34 multibacillary patients with high BIs treated for 2 years with MDT. Int $J$ Lepr, 1996; 64: 133-135.

12 Shaw IN, Rao GS, Manimozhi N et al. A long term follow up of multibacillary leprosy patients with high BI treated with WHO multibacillary regimen for a fixed duration of 2 years. Abstracts of Presentations, Asian Leprosy Congress, 9-13 November 2000, Agra, India, p. 135.

13 Gebre S, Saunderson P, Byass P. Relapses after fixed duration multiple drug therapy: the AMFES cohort. Lepr Rev, 2000; 71: 325-331.

14 World Health Organization. Chemotherapy of leprosy. WHO Technical Report Series no. 847, World Health Organization, Geneva, 1994.

15 WHO Expert Committee on Leprosy. Seventh Report. WHO Technical Report Series no. 874. World Health Organization, Geneva, 1998.

16 Croft RP, Richardus JH, Smith WC. Field treatment of acute nerve function impairment in leprosy using a standardized regimen-first year experience with 100 patients. Lepr Rev, 1997; 68: 316-325.

17 Saunderson P, Gebre S, Desta K et al. The pattern of leprosy related neuropathy in the AMFES patients in Ethiopia: definitions, incidence, risk factors and outcome. Lepr Rev, 2000; 71: 285-308.

18 Shetty VP, Ghate SD, Pai VV et al. Risk factors predisposing to relapse in leprosy: retrospective analysis of 78 $\mathrm{MB}$ and $59 \mathrm{~PB}$ cases presenting with relapse. Abstracts of Presentations, Asian Leprosy Congress, 9-13 November 2000, Agra, India, p. 136.

Bactériologie et Hygiène,

JI BAOHONG

Faculté de Médecine Pitié-Salpêtrière,

91, Boulevard de l'Hôpital,

75634 Paris Cedex 13,

France 